\title{
SIGPLAN
}

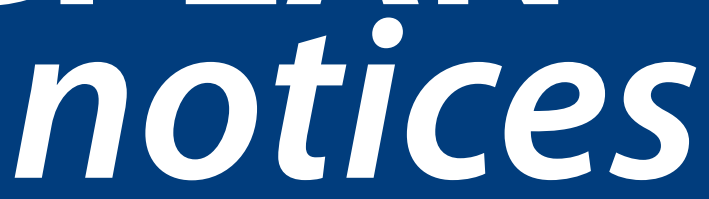

Proceedings of the 2016 ACM International Conference on Object-Oriented Programming, Systems, Languages \& Applications (OOPSLA'16) 
ACM SIGPLAN Notices

A monthly publication of ACM SIGPLAN

\section{Publications Office}

ACM, 2 Penn Plaza, Suite 701, New York, NY 10121-0701, USA

$+1-212-869-7440$

$F A X+1-212-302-9618$

ACM SIGPLAN Notices is an informal monthly publication of the Special Interest Group on Programming Languages (SIGPLAN) of ACM.

Membership in SIGPLAN is open to ACM Members or associate members for $\$ 25$ (online) and $\$ 130$ (print). Membership for ACM Student members is $\$ 15$ (online) and $\$ 130$ (print). SIGPLAN Notices is available online through the ACM Digital Library. Print members receive a hardcopy of each issue. All members receive the annual SIGPLAN CD, discounts at SIGPLAN events, and may vote in the Group's elections. ACM members of SIGPLAN may serve as officers of the Group.

Institutional or Library subscriptions to ACM SIGPLAN Notices are available for $\$ 110$ per year, and the regular back issues of the Notices may be purchased for $\$ 19$ per copy from ACM Headquarters.

Periodicals postage paid at New York, NY 10001 and at additional mailing offices.

Requests for reprints, copies of reports, or references should be sent to authors.

\section{Members Services Information: \\ acmhelp@acm.org \\ or +1-212-626-0500}

\section{Change of address:}

acmcoa@acm.org

Advertising Information:

Jennifer Ruzicka +1-212-869-7440

POSTMASTER: Send change of Address to ACM SIGPLAN Notices, ACM, 2 Penn Plaza, Suite 701, New York, NY 10121-0701.

SIGPLAN Executive Committee

SIGPLAN Sponsored Conferences

Chair

Michael Hicks

University of Maryland

mwh@cs.umd.edu

Vice Chair

Peter Thiemann

Universität Freiburg

thiemann@informatik.uni-freiburg.de

\section{Treasurer}

Cristina Lopes

University of California, Irvine

lopes@ics.uci.edu

\section{Secretary}

Kathryn S. McKinley

Microsoft Research

mckinley@microsoft.com

\section{Members at Large}

Emery Berger

University of Massachusetts Amherst

emery@cs.umass.edu

Susan Eisenbach

Imperial College, London

s.eisenbach@imperial.ac.uk

Gabriele Keller

University of New South Wales

keller@cse.unsw.edu.au

\section{Satnam Singh}

Facebook

s.singh@acm.org

Yannis Smaragdakis

University of Athens

smaragd@di.uoa.gr

\section{Past Chair}

Jan Vitek

Northeastern University

j.vitek@neu.edu

\section{Past Vice Chair}

Jeremy Gibbons

University of Oxford

jeremy.gibbons@cs.ox.ac.uk

\section{Information Director}

Matthew Fluet

Rochester Institute of Technology

mtf@cs.rit.edu

\section{SIGPLAN Program Director \\ Fran Spinola \\ spinola@hq.acm.org}

Architectural Support for Programming Languages and Operating Systems (ASPLOS)

Code Generation and Optimization (CGO)

Dynamic Languages Symposium (DLS)

Generative Programming: Concepts and Experiences (GPCE)

Haskell Symposium (HS)

International Conference on Functional Programming (ICFP)

International Symposium on Memory Management (ISMM)

Languages, Compilers, and Tools for Embedded Systems (LCTES)

Object-oriented Programming, Systems, Languages, and Applications (OOPSLA)

Onward!

Principles and Practices of Parallel Programming (PPOPP)

Principles of Programming Languages (POPL)

Programming Language Design and Implementation (PLDI)

Software Language Engineering (SLE)

Systems, Programming, Languages and Applications: Software for Humanity (SPLASH)

Virtual Execution Environments (VEE)

SIGPLAN Online
sigplan.org
facebook.com/SIGPLAN
twitter.com/sigplan

SIGPLAN Mission Statement

SIGPLAN, the premier programming languages organization, promotes awareness and advancement of research and practice in programming languages. SIGPLAN accomplishes its mission by sponsoring conferences and publishing newsletters. The SIG also serves as a coordinating body for the volunteers who wish to undertake projects to further the goals of the SIG. 
November 2-4, 2016

Amsterdam, Netherlands

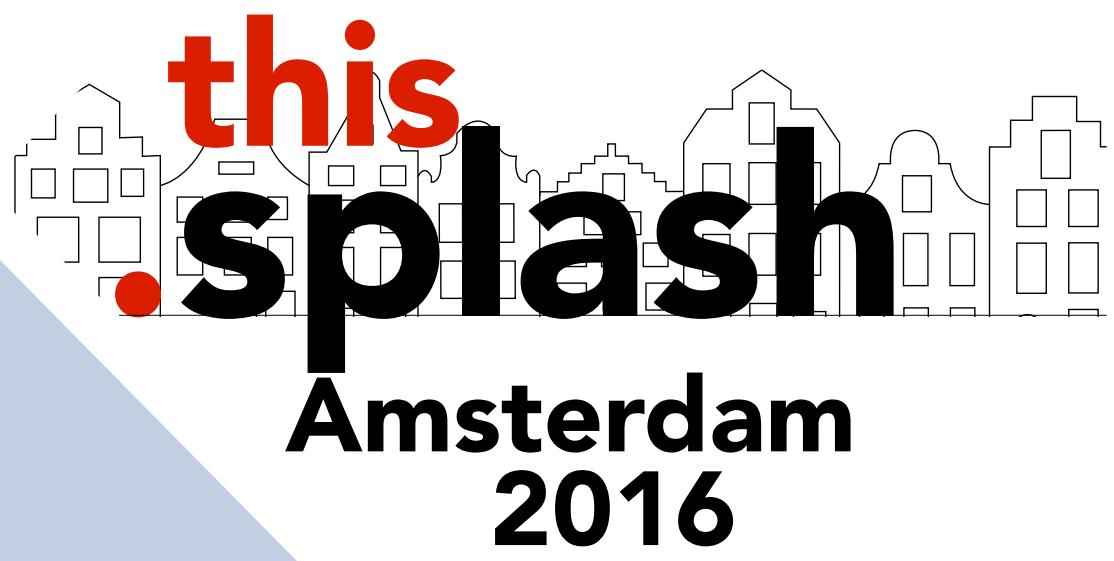

\section{OOPSLA'16}

Proceedings of the 2016 ACM SIGPLAN International Conference on

\section{Object-Oriented Programming, Systems, Languages, and Applications}

Edited by:

Eelco Visser and Yannis Smaragdakis

Sponsored by:

ACM SIGPLAN

Supported by:

LogicBlox, Universal Robots, Oracle, TU Delft, Huawei, Facebook, IBM Research, Google, itemis, ING 


\section{The Association for Computing Machinery, Inc. 2 Penn Plaza, Suite 701 New York, NY 10121-0701}

Copyright (C) 2016 by the Association for Computing Machinery, Inc (ACM). Permission to make digital or hard copies of portions of this work for personal or classroom use is granted without fee provided that the copies are not made or distributed for profit or commercial advantage and that copies bear this notice and the full citation on the first page. Copyrights for components of this work owned by others than ACM must be honored. Abstracting with credit is permitted.

To copy otherwise, to republish, to post on servers or to redistribute to lists, requires prior specific permission and/or a fee. Request permission to republish from: Publications Dept. ACM, Inc.

Fax +1-212-869-0481 or E-mail permissions@acm.org.

For other copying of articles that carry a code at the bottom of the first or last page, copying is permitted provided that the per-copy fee indicated in the code is paid through the Copyright Clearance Center, 222 Rosewood Drive, Danvers, MA 01923.

\section{Notice to Past Authors of ACM-Published Articles}

ACM intends to create a complete electronic archive of all articles and/or other material previously published by ACM. If you have written a work that was previously published by ACM in any journal or conference proceedings prior to 1978, or any SIG Newsletter at any time, and you do NOT want this work to appear in the ACM Digital Library, please inform permissions@acm.org, stating the title of the work, the author(s), and where and when published.

ACM ISBN: 978-1-4503-4444-9

Additional copies may be ordered prepaid from:

$\begin{array}{ll}\text { ACM Order Department } & \text { (U.S.A. and Canada) } \\ \text { P.O. BOX 11405 } & +1-212-626-0500 \\ \text { Church Street Station } & \text { (All other countries) } \\ \text { New York, NY 10286-1405 } & \text { Fax: +1-212-944-1318 } \\ & \text { E-mail: acmhelp@acm.org }\end{array}$

SPLASH 2016 logo designed by Tijs van der Storm

Production: Conference Publishing Consulting

D-94034 Passau, Germany, info@conference-publishing.com 


\section{Message from the Chairs}

Welcome to the 2016 ACM SIGPLAN Conference on Object-Oriented Programming, Systems, Languages, and Applications (OOPSLA). The selection of papers in this year's conference showcases the breadth and depth of research in programming languages and software development. Of the 30 paper topics listed on the submission form, the top-5 for accepted papers have been "language design", "language implementation", "concurrency", "program analysis", and "tools". Closely following are "compilers", "programming models and paradigms", and "type systems and logics". These topics are an excellent reflection of the mix of subjects that OOPSLA treats, as well as the conference's flavor.

This is the fourth year in which OOPSLA followed a two-phase selection process and the second year of double-blind reviewing. Papers advanced to the second round with specific required revisions, and were accepted only upon completion of the revisions. The revision period was 7 weeks long, giving the authors time to significantly update their papers.

The Program Committee worked hard to offer detailed reviews and make highly informed judgments. We had a very productive meeting, graciously hosted by TU Delft, with all PC members physically present. Additionally, the Program Committee carefully considered the meaning and scope of a second-phase revision. We decided on the general principle that second-phase revisions are akin to a major revision phase of a journal, for revisions that can be reasonably expected to be completed in 6-7 weeks. The required revisions could mandate new research (e.g., new experiments, case studies, or proofs) but only if one of three criteria was met: either a) there was high confidence that the risk is low, i.e., that the outcome of the new research would validate the paper's approach; or b) the committee was willing to accept the paper even if the outcome were negative for the paper's approach; or c) the author response itself had made claims that required the new research for validation.

203 papers were submitted in total. Of these, 52 were accepted and appear in these proceedings, for an acceptance rate of 25.6\%. 22 papers were co-authored by PC members, and were exclusively reviewed (and discussed separately) by members of the External Program Committee. 6 of these papers were eventually accepted $(27.3 \%)$.

We were honored to serve as Chairs of SPLASH/OOPSLA 2016. We are deeply thankful to the many people who contributed to this volume, from the authors of all papers, to the hard-working, consummately professional reviewers, and to the entire organizing committee.

Yannis Smaragdakis

OOPSLA 2016 Program Chair

Eelco Visser

SPLASH 2016 General Chair 


\section{Message from the OOPSLA Artifact Evaluation Chairs}

The goal of the OOPSLA Artifacts Evaluation process is to encourage authors of accepted OOPSLA papers to provide the artifacts associated with their papers, so future researchers can more effectively build on, and compare with, previous work. The process is implemented by having a committee, whose mission is to provide feedback to authors on how their artifact can be improved, and to assess how the artifacts support the claims of the paper. Artifact submission is voluntary and open to papers that have been conditionally accepted to the conference. The process was run independent of the technical program committee, and thus had no influence on the decision of that committee.

Papers that are viewed as meeting the claims of the paper receive a seal of approval printed on the first page of their paper. These authors are encouraged to make their artifacts publicly available upon publication of the proceedings, by including them as "source materials" in the ACM Digital Library.

Of the 52 accepted papers in the conference, 28 submitted artifacts for evaluation by this committee. Each artifact was assigned to 3 members of the committee. Each reviewer was asked to perform an initial "kicking the tires" review of the artifact's "Getting Started" guides and to build the artifact to ensure that there were no simple oversights by the authors (such as a missing file) that could be easily corrected with an interaction with the authors. After this interaction, the process continued without any other interaction with the authors. The 3 reviewers performed a more thorough review of the artifact, spending at least a full day on each artifact.

Reviewers were asked to play the role of a future user who wishes to understand the artifact, such as

- a competitive researcher who wants to compare the results of their technique in a detailed way;

- a researcher who wants to build on the research, possibly re-using it as a black box; and

- a developer who is considering implementing the idea in the paper, but first wants to validate the results produced by the artifact in their environment.

To help best simulate a future user, we chose a committee of 35 graduate students. These committee members were mostly senior $\mathrm{PhD}$ students with experience publishing papers and creating artifacts. Each artifact was judged on the following dimensions: consistency with the paper, completeness of the artifact, documentation, and ease of reuse. After submitting a review, the 3 reviewers had a detailed online discussion of the artifact and came to a consensus on a final decision for the artifact.

Of the 28 submitted artifacts, 24 met the expectations set in the paper, and thus, received the seal for their paper. Three of these artifacts were recognized as Distinguished Artifacts and were announced at the conference. We hope that these 24 papers will be recognized not only for their excellent technical contribution, but also for the quality of their artifact. We hope that many of the accepted authors will make available their artifacts along with the paper in the ACM Digital Library.

We would like to thank the committee for their efforts, detailed feedback to the authors, and substantial time commitments during the busy summer months. We also want to thank the authors of the 28 papers for preparing their artifacts. We feel this process is an important step towards increasing reproducibility in our field.

Michael Bond and Michael Hind

2016 OOPSLA Artifacts Co-chairs 


\section{OOPSLA 2016 Organization}

\section{Organizing Committee}

\section{General Chair}

Eelco Visser

\section{Program Chair}

Yannis Smaragdakis

\section{Artifact Evaluation Co-Chairs}

Michael Bond

Michael Hind

\section{Web and Publicity Co-Chairs}

Ronald Garcia

Tijs van der Storm

\section{Publications Chair}

Alex Potanin

Treasurer \& Conference Manager

Annabel Satin

Sponsorship Chair

Jurgen Vinju

Video Chair

Michael Hilton

\section{Student Volunteer Co-Chairs}

Daco Harkes

Giovanni Viviani

\section{Steering Committee}

Antony L. Hosking (Steering Committee Chair)

Jonathan Aldrich

Andrew Black

Patrick Eugster

Michael Hicks

Robert Hirschfeld

Shriram Krishnamurthi

Crista Lopes

Todd Millstein

Gail Murphy

Emerson Murphy-Hill

Yannis Smaragdakis

Peter Thiemann

Eelco Visser
Delft University of Technology, Netherlands

University of Athens, Greece

Ohio State University, USA

IBM Research, USA

University of British Columbia, Canada

CWI / University of Groningen, Netherlands

Victoria University of Wellington, New Zealand

Petit Canard Kitchen, UK

CWI, Netherlands

Oregon State University, USA

Delft University of Technology, Netherlands

University of British Columbia, Canada
Australian National University / Data61, Australia /

Purdue University, USA

Carnegie Mellon University, USA

Portland State University, USA

Purdue University, USA /

Technical University of Darmstadt, Germany

University of Maryland at College Park, USA

HPI, Germany

Brown University, USA

University of California at Irvine, USA

University of California at Los Angeles, USA

University of British Columbia, Canada

Google, USA

University of Athens, Greece

University of Freiburg, Germany

Delft University of Technology, Netherlands 


\section{Program Committee}

Yannis Smaragdakis (Program Chair)
Jonathan Aldrich
Emery Berger
Gilad Bracha
Michael Carbin
Shigeru Chiba
Wei Ngan Chin
Stephen Chong
Isil Dillig
Sophia Drossopoulou
Patrick Eugster
Robby Findler
David Grove
Shan Shan Huang
Richard Jones
Milind Kulkarni
Julia Lawall
Ben Livshits
Anders Møller
James Noble
Klaus Ostermann
Jens Palsberg
Sukyoung Ryu
Caitlin Sadowski
Manu Sridharan
Olivier Tardieu
Frank Tip
Laurence Tratt
Jan Vitek
Tijs van der Storm

\section{External Review Committee}

Davide Ancona

David Bacon

Hans-J. Boehm

Michael Bond

John Boyland

Michael D. Ernst

Julian Dolby

Stephen J. Fink

Jeffrey S. Foster

Yossi Gil

Thomas Gross

Shriram Krishnamurthi

Doug Lea

Mira Mezini

Emerson Murphy-Hill
University of Athens, Greece

Carnegie Mellon University, USA

University of Massachusetts at Amherst, USA

Google, USA

MIT, USA

University of Tokyo, Japan

National University of Singapore, Singapore

Harvard University, USA

University of Texas at Austin, USA

Imperial College London, UK

Purdue University, USA

Northwestern University, USA

IBM Research, USA

Logicblox, USA

University of Kent, UK

Purdue University, USA

Inria / LIP6, France

Microsoft Research, USA

Aarhus University, Denmark

Victoria University of Wellington, New Zealand

University of Tübingen, Germany

University of California at Los Angeles, USA

KAIST, South Korea

Google, USA

Samsung Research, USA

IBM Research, USA

Samsung Research, USA

King's College London, UK

Northeastern University, USA

CWI / University of Groningen, Netherlands
University of Genova, Italy

Google, USA

Google, USA

Ohio State University, USA

University of Wisconsin-Milwaukee, USA

University of Washington, USA

IBM Research, USA

IBM Research, USA

University of Maryland at College Park, USA

Technion, Israel

ETH Zurich, Switzerland

Brown University, USA

SUNY Oswego, USA

TU Darmstadt, Germany

Google, USA 
Bruno C. d. S. Oliveira

Erez Petrank

Bill Pugh

Jeremy Siek

Éric Tanter

Eli Tilevich

Martin Vechev

Tobias Wrigstad
University of Hong Kong, China

Technion, Israel

University of Maryland at College Park, USA

Indiana University, USA

University of Chile, Chile

Virginia Tech, USA

ETH Zurich, Switzerland

Uppsala University, Sweden

\section{External Reviewers}

Michael Adam
John Altidor
Rajeev Alur
Thanassis Avgerinos
Mark Batty
Aggelos Biboudis
Sebastian Burckhardt
Zhuohong Cai
Luke Cheeseman
Sylvan Clebsch
Andreea Costea
Jácome Cunha
Eelco Dolstra
Alastair Donaldson
Susan Eisenbach
Sebastian Erdweg
Erik Ernst
Spencer Florence

Juliana Franco
Arjun Guha
Kyle Hale
Mark Harman
Felienne Hermans
Baris Kasikci
Paul Kelly
Quang Loc Le
Ton Chanh Le
Angelina Lee
Benedict Lee
Hendrik Lensch
Zaki Malik
Valentina Manea
Aristeidis Mastoras
Alexander Matveev
Pat Nicholson
Ganesan Ramalingam

\author{
John Regehr \\ Tiark Rompf \\ Adrian Sampson \\ Ulrik Pagh Schultz \\ Rahul Sharma \\ George Steed \\ Charles Sutton \\ Quang-Trung Ta \\ Luca Della Toffola \\ Muoi Tran \\ Viktor Vafeiadis \\ Benoit Valiron \\ Chao Wang \\ John Wickerson \\ Tim Wood \\ Eric Van Wyk \\ Cristian Zamfir \\ Edgardo Zoppi
}

\section{Artifacts Evaluation Committee}

\author{
Michael Bond (Artifact Evaluation Co-Chair) \\ Michael Hind (Artifact Evaluation Co-Chair) \\ Rohan Achar \\ Shoaib Akram \\ Esben Andreasen \\ Tanzirul Azim \\ Jonathan Bell \\ Stephan Brandauer \\ Elias Castegren \\ Sylvan Clebsch \\ Karl Cronburg \\ Will Dietz \\ Jonathan Eyolfson \\ Francisco Ferreira \\ Ben Greenman \\ Yongjian $\mathrm{Hu}$ \\ Timothy Jones
}

Ohio State University, USA

IBM Research, USA

University of California at Irvine, USA

Ghent University, Belgium

Aarhus University, Denmark

University of California at Riverside, USA

Columbia University, USA

Uppsala University, Sweden

Uppsala University, Sweden

Imperial College London, UK

Tufts University, USA

University of Illinois at Urbana-Champaign, USA

University of Waterloo, Canada

McGill University, Canada

Northeastern University, USA

University of California at Riverside, USA

Victoria University of Wellington, New Zealand 
Wing Lam

Ravi Mangal

Jabier Martinez

Luis Mastrangelo

Diogenes Nunez

Changhee Park

Luís Pina

Christoffer Quist Adamsen

Cosmin Radoi

Marianna Rapoport

Brianna M. Ren

Malavika Samak

Jiasi Shen

John L. Singleton

Justin Smith

Ganesha Upadhyaya

James R. Wilcox

Wei Yang

Xi Yang

Xin Zhang
University of Illinois at Urbana-Champaign, USA

Georgia Institute of Technology, USA

University of Luxembourg, Luxembourg

University of Lugano, Switzerland

Tufts University, USA

KAIST, South Korea

Imperial College London, UK

Aarhus University, Denmark

University of Illinois at Urbana-Champaign, USA

University of Waterloo, Canada

University of Maryland at College Park, USA

Indian Institute of Science Bangalore, India

Massachusetts Institute of Technology, USA

University of Central Florida, USA

North Carolina State University, USA

Iowa State University, USA

University of Washington, USA

University of Illinois at Urbana-Champaign, USA

Australian National University, Australia

Georgia Institute of Technology, USA 


\section{SPLASH 2016 Sponsors and Supporters}

Sponsors:

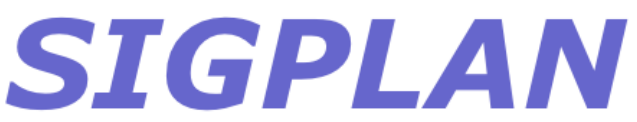

Gold Supporters:

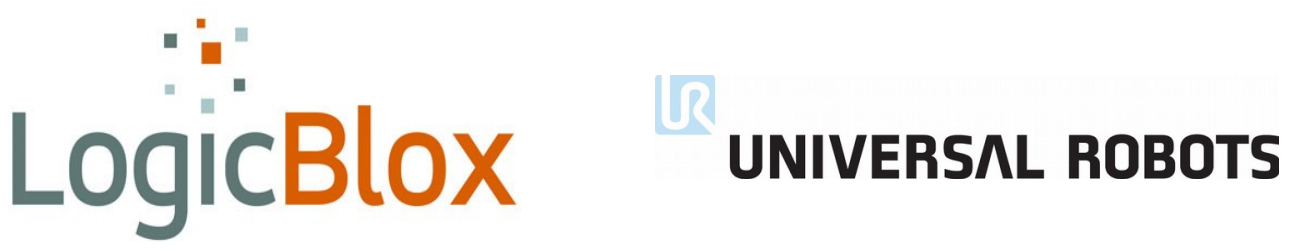

Silver Supporters:
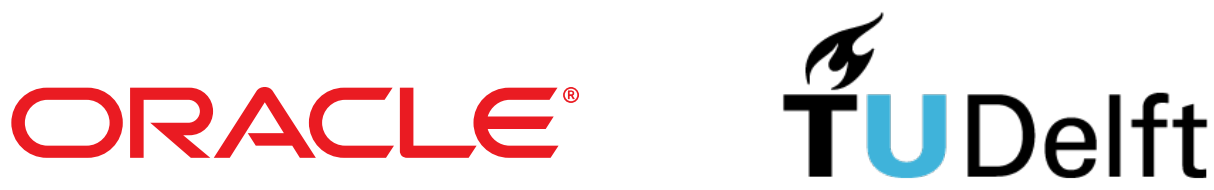

Bronze Supporters:

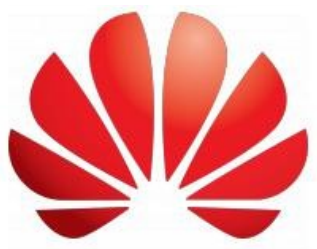

\section{facebook}

\section{HUAWEI}

IBM Research
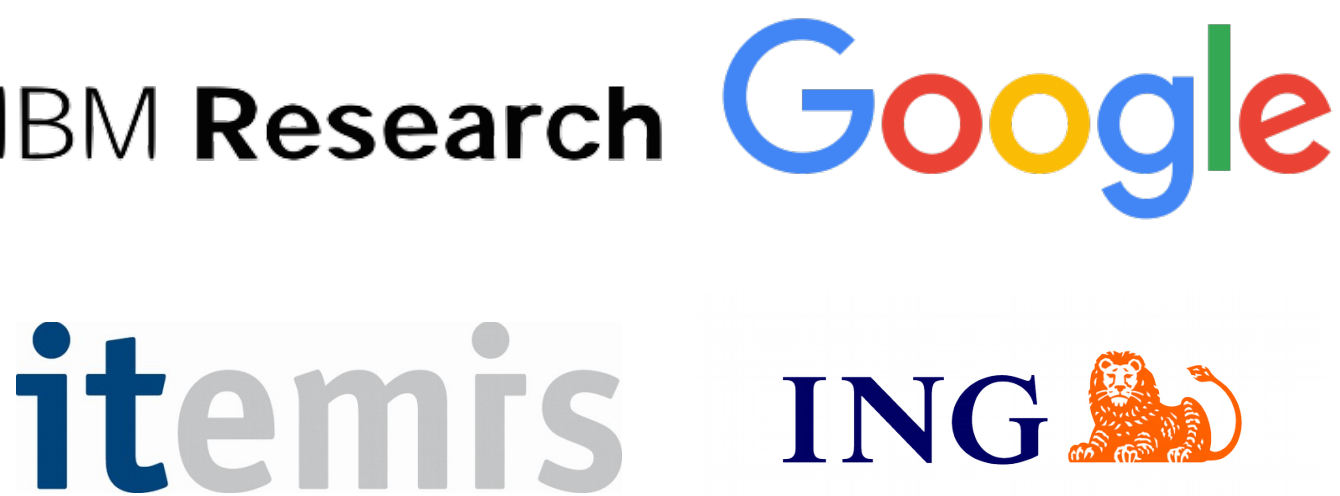

ING

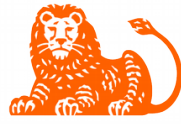




\section{Contents}

\section{Frontmatter}

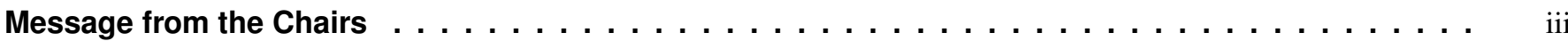

Message from the OOPSLA Artifact Evaluation Chairs $\ldots \ldots \ldots \ldots \ldots \ldots$ iv

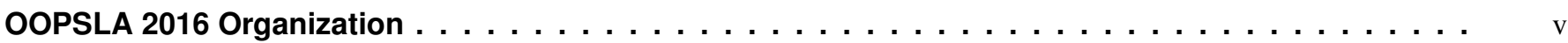

SPLASH 2016 Sponsors and Supporters $\ldots \ldots \ldots \ldots \ldots \ldots \ldots \ldots \ldots \ldots \ldots$ ix $\ldots \ldots \ldots \ldots$

\section{Optimization and Performance}

A Compiler for Throughput Optimization of Graph Algorithms on GPUs

Sreepathi Pai and Keshav Pingali — University of Texas at Austin, USA . . . . . . . . . . . . . . . 1

Automatic Parallelization of Pure Method Calls via Conditional Future Synthesis

Rishi Surendran and Vivek Sarkar — Rice University, USA . . . . . . . . . . . . . . . . . . . . .

Portable Inter-workgroup Barrier Synchronisation for GPUs

Tyler Sorensen, Alastair F. Donaldson, Mark Batty, Ganesh Gopalakrishnan, and Zvonimir Rakamarić — Imperial

College London, UK; University of Kent, UK; University of Utah, USA . . . . . . . . . . . . . . . . .

Parallel Incremental Whole-Program Optimizations for Scala.js

Sébastien Doeraene and Tobias Schlatter —EPFL, Switzerland . . . . . . . . . . . . . . . . .

\section{Semantics and Verification}

Semantics-Based Program Verifiers for All Languages

Andrei Stefănescu, Daejun Park, Shijiao Yuwen, Yilong Li, and Grigore Roşu - University of Illinois at Urbana-

Champaign, USA, Runtime Verification, USA . . . . . . . . . . . . . . . . . . . . . . . .

Hoare-Style Specifications as Correctness Conditions for Non-linearizable Concurrent Objects

Ilya Sergey, Aleksandar Nanevski, Anindya Banerjee, and Germán Andrés Delbianco - University College London, UK;

IMDEA Software Institute, Spain . . . . . . . . . . . . . . . . . . . . . . . . . . .

An Operational Semantics for $\mathbf{C} / \mathbf{C}_{++11}$ Concurrency

Kyndylan Nienhuis, Kayvan Memarian, and Peter Sewell — University of Cambridge, UK . . . . . . . . . .

Modeling and Analysis of Remote Memory Access Programming

Andrei Marian Dan, Patrick Lam, Torsten Hoefler, and Martin Vechev - ETH Zurich, Switzerland; University of

Waterloo, Canada . . . . . . . . . . . . . . . . . . . . . . . . . . .

\section{Program Synthesis}

Deriving Divide-and-Conquer Dynamic Programming Algorithms using Solver-Aided Transformations Shachar Itzhaky, Rohit Singh, Armando Solar-Lezama, Kuat Yessenov, Yongquan Lu, Charles Leiserson, and Rezaul Chowdhury — Massachusetts Institute of Technology, USA; Stony Brook University, USA . . . . . . . . . . .

Speeding Up Machine-Code Synthesis

Venkatesh Srinivasan, Tushar Sharma, and Thomas Reps - University of Wisconsin-Madison, USA . . . . . . .

Automated Reasoning for Web Page Layout

Pavel Panchekha and Emina Torlak — University of Washington, USA . . . . . . . . . . . . .

FIDEX: Filtering Spreadsheet Data using Examples

Xinyu Wang, Sumit Gulwani, and Rishabh Singh - University of Texas at Austin, USA; Microsoft Research, USA . .

\section{Language Design and Programming Models I}

\section{Extensible Access Control with Authorization Contracts}

Scott Moore, Christos Dimoulas, Robert Bruce Findler, Matthew Flatt, and Stephen Chong - Harvard University, USA; 
Gentrification Gone too Far? Affordable 2nd-Class Values for Fun and (Co-)Effect

Leo Osvald, Grégory Essertel, Xilun Wu, Lilliam I. González Alayón, and Tiark Rompf — Purdue University, USA .

Incremental Forest: A DSL for Efficiently Managing Filestores

Jonathan DiLorenzo, Richard Zhang, Erin Menzies, Kathleen Fisher, and Nate Foster - Cornell University, USA;

University of Pennsylvania, USA; Tufts University, USA . . . . . . . . . . . . . . . . . . . . .

LaCasa: Lightweight Affinity and Object Capabilities in Scala

Philipp Haller and Alex Loiko — KTH, Sweden; Google, Sweden . . . . . . . . . . . . . . . . . .

\section{Programming Frameworks, Tools, and Methodologies}

Purposes, Concepts, Misfits, and a Redesign of Git

Santiago Perez De Rosso and Daniel Jackson — Massachusetts Institute of Technology, USA . . . . . . . . . .

Apex: Automatic Programming Assignment Error Explanation

Dohyeong Kim, Yonghwi Kwon, Peng Liu, I. Luk Kim, David Mitchel Perry, Xiangyu Zhang, and Gustavo Rodriguez-

Rivera — Purdue University, USA . . . . . . . . . . . . . . . . . . . . . . . .

Asserting Reliable Convergence for Configuration Management Scripts

Oliver Hanappi, Waldemar Hummer, and Schahram Dustdar - Vienna University of Technology, Austria . . . . . .

Dependent Partitioning

Sean Treichler, Michael Bauer, Rahul Sharma, Elliott Slaughter, and Alex Aiken — Stanford University, USA; NVIDIA

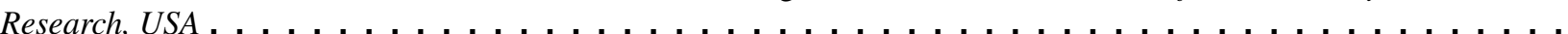

\section{Static Analysis}

Accelerating Program Analyses by Cross-Program Training

Sulekha Kulkarni, Ravi Mangal, Xin Zhang, and Mayur Naik — Georgia Tech, USA . . . . . . . . . . .

An Improved Algorithm for Slicing Machine Code

Venkatesh Srinivasan and Thomas Reps - University of Wisconsin-Madison, USA . . . . . . . . . . . .

Call Graphs for Languages with Parametric Polymorphism

Dmitry Petrashko, Vlad Ureche, Ondřej Lhoták, and Martin Odersky — EPFL, Switzerland; University of Waterloo,

Canada . . . . . . . . . . . . . . . . . . . . . . . . .

Type Inference for Static Compilation of JavaScript

Satish Chandra, Colin S. Gordon, Jean-Baptiste Jeannin, Cole Schlesinger, Manu Sridharan, Frank Tip, and Youngil Choi - Samsung Research, USA; Drexel University, USA; Northeastern University, USA; Samsung Electronics, South Korea

\section{Concurrency Analysis and Model Checking}

\section{Directed Synthesis of Failing Concurrent Executions}

Malavika Samak, Omer Tripp, and Murali Krishna Ramanathan — IISc Bangalore, India; Google, USA . . . . . . .

Maximal Causality Reduction for TSO and PSO

Shiyou Huang and Jeff Huang — Texas A\&M University, USA . . . . . . . . . . . . . . . . . . . . . . . 447

Precise and Maximal Race Detection from Incomplete Traces

Jeff Huang and Arun K. Rajagopalan — Texas A\&M University, USA . . . . . . . . . . . . . . . . . . 462

Stateless Model Checking with Data-Race Preemption Points

Ben Blum and Garth Gibson - Carnegie Mellon University, USA ． . . . . . . . . . . . . . . . .

\section{Language Design and Programming Models II}

\section{Automatic Enforcement of Expressive Security Policies using Enclaves}

Anitha Gollamudi and Stephen Chong — Harvard University, USA . . . . . . . . . . . . . . . .

Chain: Tasks and Channels for Reliable Intermittent Programs

Alexei Colin and Brandon Lucia - Carnegie Mellon University, USA

GEMs: Shared-Memory Parallel Programming for Node.js

Daniele Bonetta, Luca Salucci, Stefan Marr, and Walter Binder - Oracle Labs, Austria; University of Lugano, Switzer-

land; JKU Linz, Austria . . . . . . . . . . . . . . . . . . . . . . . . . . . . . . . 


\section{OrcO: A Concurrency-First Approach to Objects}

Arthur Michener Peters, David Kitchin, John A. Thywissen, and William R. Cook - University of Texas at Austin, USA;

Google, USA . . . . . . . . . . . . . . . . . . . . . . . . .

\section{Principles, Across the Compilation Stack}

\section{Semantic Subtyping for Imperative Object-Oriented Languages}

Davide Ancona and Andrea Corradi — University of Genoa, Italy ～. . . . . . . . . . . . . . . . . . . . . 568

Parsing with First-Class Derivatives

Jonathan Immanuel Brachthäuser, Tillmann Rendel, and Klaus Ostermann — University of Tübingen, Germany . . .

The Missing Link: Explaining ELF Static Linking, Semantically

Stephen Kell, Dominic P. Mulligan, and Peter Sewell — University of Cambridge, UK . . . . . . . . . . . .

Type Soundness for Dependent Object Types (DOT)

Tiark Rompf and Nada Amin — Purdue University, USA; EPFL, Switzerland . . . . . . . . . . . .

\section{Runtime Support}

Efficient and Thread-Safe Objects for Dynamically-Typed Languages

Benoit Daloze, Stefan Marr, Daniele Bonetta, and Hanspeter Mössenböck - JKU Linz, Austria; Oracle Labs, Austria

Hybrid STM/HTM for Nested Transactions on OpenJDK

Keith Chapman, Antony L. Hosking, and J. Eliot B. Moss - Purdue University, USA; Australian National University,

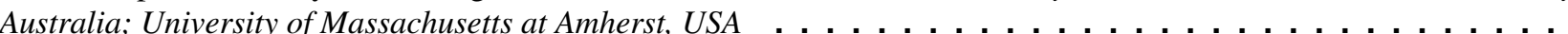

Makalu: Fast Recoverable Allocation of Non-volatile Memory

Kumud Bhandari, Dhruva R. Chakrabarti, and Hans-J. Boehm - Rice University, USA; Hewlett Packard Labs, USA;

Google, USA . . . . . . . . . . . . . . . . . . . . . . . . . . .

Prioritized Garbage Collection: Explicit GC Support for Software Caches

Diogenes Nunez, Samuel Z. Guyer, and Emery D. Berger - Tufts University, USA; University of Massachusetts at

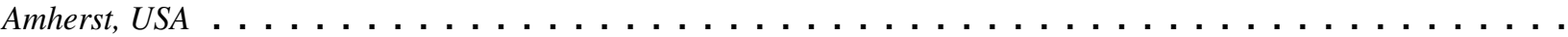

\section{Program Modeling and Learning}

Computing Repair Alternatives for Malformed Programs using Constraint Attribute Grammars

Friedrich Steimann, Jörg Hagemann, and Bastian Ulke — Fernuniversität in Hagen, Germany . . . . . . . . . .

Probabilistic Model for Code with Decision Trees

Veselin Raychev, Pavol Bielik, and Martin Vechev — ETH Zurich, Switzerland . . . . . . . . . . . . .

Ringer: Web Automation by Demonstration

Shaon Barman, Sarah Chasins, Rastislav Bodik, and Sumit Gulwani - University of California at Berkeley, USA;

University of Washington, USA; Microsoft Research, USA . . . . . . . . . . . . . . . . . . . . . . .

Scalable Verification of Border Gateway Protocol Configurations with an SMT Solver

Konstantin Weitz, Doug Woos, Emina Torlak, Michael D. Ernst, Arvind Krishnamurthy, and Zachary Tatlock — University of Washington, USA

\section{Typing, in Practice}

\section{A Practical Framework for Type Inference Error Explanation}

Calvin Loncaric, Satish Chandra, Cole Schlesinger, and Manu Sridharan - University of Washington, USA; Samsung

Research, USA . . . . . . . . . . . . . . . . . . . . . . . .

Dynamically Diagnosing Type Errors in Unsafe Code

Stephen Kell - University of Cambridge, UK

First-Class Effect Reflection for Effect-Guided Programming

Yuheng Long, Yu David Liu, and Hridesh Rajan — Iowa State University, USA; SUNY Binghamton, USA . . . . .

Java and Scala's Type Systems are Unsound: The Existential Crisis of Null Pointers

Nada Amin and Ross Tate — EPFL, Switzerland; Cornell University, USA . . . . . . . . . . . . . . . . 


\section{Bug Detection Analysis and Model Checking}

Finding Compiler Bugs via Live Code Mutation

Chengnian Sun, Vu Le, and Zhendong Su — University of California at Davis, USA . . . . . . . . . . . .

Finding Resume and Restart Errors in Android Applications

Zhiyong Shan, Tanzirul Azim, and Iulian Neamtiu - University of Central Missouri, USA; University of California at Riverside, USA; New Jersey Institute of Technology, USA . . . . . . . . . . . . . . . . . . . . . . . . . .

Low-Overhead and Fully Automated Statistical Debugging with Abstraction Refinement

Zhiqiang Zuo, Lu Fang, Siau-Cheng Khoo, Guoqing Xu, and Shan Lu - University of California at Irvine, USA; National University of Singapore, Singapore; University of Chicago, USA . . . . . . . . . . . . . .

To Be Precise: Regression Aware Debugging

Rohan Bavishi, Awanish Pandey, and Subhajit Roy — IIT Kanpur, India . . . . . . . . . . . . . . . .

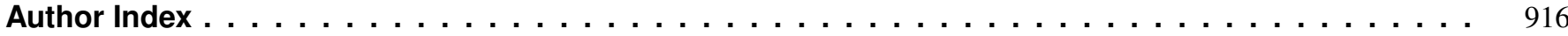

